\title{
REVISIÓN
}

\section{Efecto de los probióticos en el control de la obesidad en humanos: hipótesis no demostradas}

\author{
Amparo Arias ${ }^{a, *}$ y Núria Mach ${ }^{a, b}$ \\ aÀrea de Ciències de la Salut, Institut Internacional de Postgrau, Universitat Oberta de Catalunya, Barcelona, España \\ 'INRA, Département de Génétique Animale et Biologie Intégrative, J ouy-en-J osas, Francia
}

Recibido el 19 de septiembre de 2011; aceptado el 7 de junio de 2012

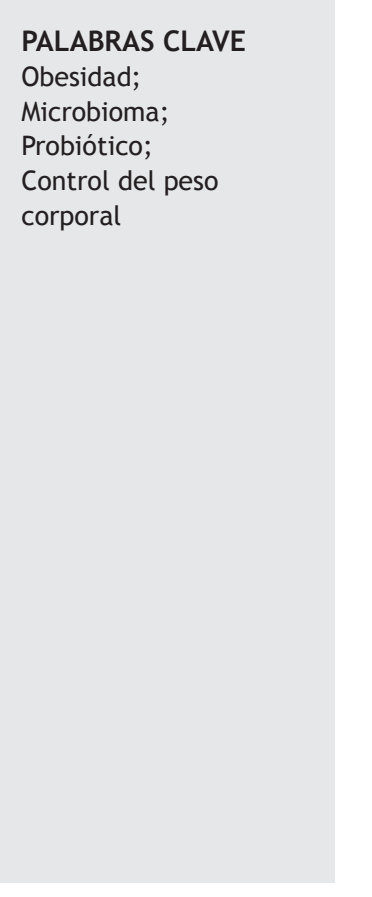

\begin{abstract}
Resumen
El presente trabajo tiene como objetivo esclarecer y contrastar las diferentes publicaciones que asocian los probióticos con la obesidad, además de profundizar en el papel del microbioma humano en enfermedades como la obesidad. Se realizó una extensa búsqueda de publicaciones científicas (estudios en modelos animales, celulares, ensayos clínicos y revisiones) en las bases de datos electrónicas especializadas PubMed central-NCBI, Elsevier Journal, Scielo España, Scirus y Science Direct para establecer el estado actual del potencial efecto de los probióticos en el control de la obesidad de las personas, así como la relación entre microbioma y obesidad. La microbiota intestinal y los probióticos producen un efecto positivo para la salud regulando las funciones inmunológicas del individuo y protegiéndolo de infecciones y procesos de inflamación crónica. Sin embargo, actualmente hay estudios con resultados dispares, que señalan (pero no confirman) a la microbiota intestinal como un nuevo factor que podría estar implicado en la regulación del peso corporal y las enfermedades asociadas a la obesidad. El Proyecto MetaHIT ha encontrado tres géneros de población genética en el microbioma intestinal humano, y dos de ellos (Bacteroides y Ruminococcus) podrían estar relacionados con la regulación del peso corporal de su hospedador. A pesar de que las relaciones causa-efecto entre la microbiota intestinal y la obesidad no están todavía bien establecidas, la manipulación intencionada de la microbiota intestinal a través de la dieta se propone como una posible nueva herramienta para prevenir o modificar el riesgo de obesidad y, en particular, las enfermedades metabólicas asociadas a esta. Se requieren más estudios clínicos en humanos para esclarecer el posible efecto que tienen los probióticos en el control del peso corporal.
\end{abstract}

(C) 2011 Asociación Española de Dietistas-Nutricionistas. Publicado por Elsevier España, S.L. Todos los derechos reservados.

\footnotetext{
*Autor para correspondencia.

Correo electrónico: amparoariasp@hotmail.com (A. Arias Pineda).
} 


\section{KEYWORDS}

Obesity; Metagenome;

Probiotic;

Body weight control

\section{Effects of Probiotics on Human Obesity Control: An Unproven Hypothesis}

\section{Abstract}

The objective of the present study is to review the different publications associated with probiotics and obesity, as well as to get some new insights regarding the role of the microbiome in diseases such as obesity. An extensive search for scientific publications (studies in animal models, cells, clinical trials and reviews) was performed in the following specialist computer databases (PubMed central (PMC)-NCBI, Elsevier Journal, Scielo Spain, Scirus, Science Direct) to establish the current status of the potential effect of probiotics in the control of obesity in humans, as well as the relationship between intestinal microbiota and obesity. The intestinal microbiota and oral probiotics have a positive effect on human health, as they can regulate the immune functions and protect from infections and chronic inflammatory processes. Although divergent results have recently been reported, it has been shown but not confirmed that intestinal microbiota might play a role as a new factor associated with the regulation of body weight and obesity-related diseases. The international MetaHIT project has shown that human microbiome populations can be grouped into three different enterotypes. Two of these enterotypes (Bacteroides and Ruminococcus) seem to encode functions related to obesity. Although the relationship between intestinal microbiota and obesity are not yet well established, the attempt to manipulate intestinal microbiota through diet is suggested as a new plausible approach to prevent, or modify the risk of, obesity and its related diseases. More clinical studies in humans are required to clarify the possible effect of probiotics on obesity.

(c) 2011 Asociación Española de Dietistas-Nutricionistas. Published by Elsevier España, S.L. All rights reserved.

\section{Introducción}

La epidemia de obesidad es uno de los principales desafíos sanitarios del mundo ${ }^{1}$. Actualmente hay estudios que indican una conexión entre los probióticos y la obesidad, particularmente entre probióticos que contienen firmicutes, en particular Lactobacillus spp. ${ }^{2}$. Los probióticos son alimentos que contienen microorganismos vivos que, ingeridos en cantidades suficientes, producen efectos positivos para la salud por su acción directa e indirecta mediante interacciones con la microflora intestinal. Hoy se puee investigar la distribución y la cuantificación filogenética de la microflora intestinal, así como sus funciones biológicas, mediante las tecnologías high-throughput basadas en la secuenciación del ADN o el ARN microbiano. Describir la composición de la microbiota intestinal, así como su función biológica y su relación con posibles enfermedades como la obesidad en humanos, es de gran interés. De hecho, el proyecto MetaHIT (2008-2012) tiene como objetivo establecer asociaciones entre el microbioma intestinal de los humanos y el estado de salud de las personas. Recientemente se ha publicado un estudio que muestra que los humanos tenemos tres tipos de enterotipos bacterianos y además apunta a que es posible que los individuos del enterotipo 1 en los que predomina el género Bacteroides (género especializado en la degradación de los hidratos de carbono) tienen menos problemas de obesidad, y que los del enterotipo 3, en quienes el género dominante es Ruminococcus (especializado en absorber azúcares), pueden presentar problemas de sobrepeso ${ }^{3}$. Hasta el momento, no existe relación entre el segundo enterotipo, caracterizado por ser rico en Prevotella y Desulfovibrio (estas bacterias actúan sinérgicamente para degradar las glucoproteínas de mucina presentes en la capa mucosa del intestino) y la obesidad. El consorcio europeo, con Dinamarca como eje investigador en la obesidad, y el Dr. Francisco Guarner, responsable del proyecto en España, están llevando a cabo una serie de investigaciones relacionadas con ciertas afecciones del aparato intestinal, y han presentado los primeros resultados que comparan el microbioma intestinal de las personas obesas y el de las personas que tienen un índice de masa corporal (IMC) considerado de normopeso $(18,5-24,9)$ para averiguar si dicha diferencia en el tipo de bacterias puede ser la causa de que ciertos individuos y no otros tengan propensión a aumentar de peso. No obstante, son pocos aún los estudios realizados sobre la relación entre los probióticos, la microbiota intestinal y la obesidad en humanos. Además, los resultados son aún dispares y heterogéneos, lo que contribuye a crear confusión entre la asociación de la ingesta de probióticos y la reducción del riesgo de sufrir obesidad. Así pues, hay necesidad de contrastar los datos publicados y esclarecer la relación entre los probióticos y el riesgo de obesidad. En esta revisión se pretende describir, desde la ciencia actual, la relación entre los probióticos, el microbioma intestinal y el control del peso de los humanos, postulando que los probióticos, como suplementos alimenticios, podrían ser utilizados para beneficiar el estado de salud de la población. 


\section{Obesidad}

Se define como la acumulación excesiva de grasa corporal. En España se ha descrito un aumento en la prevalencia de sobrepeso y obesidad ${ }^{4}$, en población tanto adulta como infanto-juvenil. Según la última Encuesta Nacional de Salud ${ }^{5}$ (ENS 2006; Ministerio de Sanidad y Política Social), con datos declarados, el 37,8 y el $15,6 \%$ de los adultos españoles mayores de 18 años tenían sobrepeso y obesidad respectivamente; en la población infantil, estas cifras alcanzaron el 8,9 y el $18,7 \%$. Utilizando los resultados del estudio enKid (2005), con medidas objetivas, las prevalencias de sobrepeso y obesidad en población infanto-juvenil española (2-24 años) alcanzaron el 13,9 y $12,4 \%$ respectivamente 6 . Respecto a la carga de enfermedad de la obesidad, estudios recientes en España indican una mortalidad atribuible a la obesidad del $8,5 \%$. Se reconoce actualmente que la epidemia de obesidad, con los riesgos que conlleva para la salud, es un importante problema de salud pública y uno de los principales desafíos sanitarios del mundo desarrollado ${ }^{1}$. Las personas con obesidad central (o abdominal), que parece ser reflejo del incremento de la grasa visceral (en contraposición a la subcutánea), son las que tienen mayor riesgo. La obesidad se asocia a numerosos factores de riesgo cardiovascular como dislipemia, hipertensión, diabetes mellitus, marcadores inflamatorios y estado protrombótico ${ }^{8}$. El modelo más comúnmente aceptado para explicar la obesidad humana se basa en la interacción entre predisposición genética y factores ambientales como la vida sedentaria y una alimentación poco saludable .

\section{Probióticos}

Probiótico se define como "ingrediente alimentario microbiano vivo que, al ser ingerido en cantidades suficientes, ejerce efectos benéficos sobre la salud de quien lo consume ${ }^{10}$. arias especies de Lactobacillus y Bifidobacterium combinadas (o no) con Streptococcus thermophilus (fig. 1) son las principales bacterias usadas como probióticos en yogures y otros productos lácteos fermentados ${ }^{11,12}$. Con el fin de evaluar las propiedades de los probióticos, la onsulta de Expertos FAO OMS ${ }^{13}$ sobre "Evaluación de las Propiedades Saludables y Nutricionales de los Probióticos en los Alimentos" (incluida la leche en polvo con bacterias vivas del ácido láctico, octubre de 2001) propuso que se utilizaran las siguientes directrices: a) los microorganismos probióticos utilizados en los alimentos deberían ser capaces no sólo de sobrevivir al paso por el aparato digestivo, sino también de proliferar en el intestino; esto significa que deberían ser resistentes a los jugos gástricos y poder crecer en presencia de bilis, en las condiciones existentes en los intestinos, o ser consumidos en un alimento que, actuando como vehículo, les permita sobrevivir al paso por el estómago y la exposición a la bilis; b) los probióticos deben poder ejercer sus efectos beneficiosos en el huésped mediante su crecimiento y o actividad en el cuerpo humano ${ }^{14,15}$; sin embargo, lo que importa es la especificidad de la acción, y no la fuente del microorganismo, y c) es necesario perfeccionar los ensayos in vitro para comprobar y así garantizar la capacidad de los probióticos de funcionar en los seres humanos o realizar

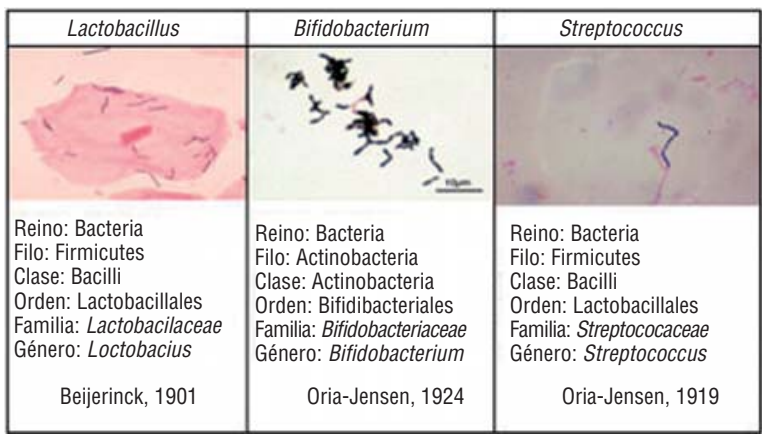

i ura Clasificación científica de los principales géneros probióticos.

ensayos clínicos en humanos que permitan estudiar directamente dicha relación (en la tabla 1 se muestran las especies más usadas) ${ }^{16}$. Pese a que se ha estudiado mucho los mecanismos biológicos de acción que relacionan los efectos de los probióticos en la disminución de la intolerancia a la lactosa y la estimulación del sistema inmunitario para reducir la incidencia o la gravedad de infecciones gastrointestinales, recientemente se está empezando a estudiar su posible papel en el control del peso corporal ${ }^{1}$.

\section{Papel potencial de los probióticos en el control del peso corporal}

En el sistema digestivo existen más de 400 especies bacterianas ${ }^{18}$; más de la mitad del peso de la materia que se encuentra en el colon corresponde a células bacterianas cuyo número es 10 veces el de las células de los tejidos que constituyen el cuerpo humano. El estómago contiene normalmente pocas bacterias (100 ufc/ml de jugo gástrico), mientras que la concentración bacteriana aumenta a lo largo del intestino hasta llegar a una concentración final en el colon de $10^{12}$ bacterias $\mathrm{g}$. La colonización bacteriana del intestino comienza con el nacimiento y continúa durante toda la vida, con cambios notables en función de la edad ${ }^{1}$ (la tabla $2^{20}$ muestra los microorganismos que se encuentran en la vía gastrointestinal humana). Describir la composición de la microbiota intestinal, así como su función biológica y su relación con posibles enfermedades como la obesidad en humanos, es de gran interés. De hecho, el proyecto MetaHIT (2008-2012) tiene como objetivo central establecer nuevas hipótesis de asociaciones entre el microbioma intestinal, la salud y la enfermedad de las personas, así como comprender los mecanismos subyacentes y las interacciones entre huésped y microorganismo. Uno de los aspectos en que se centra el estudio es el posible papel de los hallazgos en la obesidad, tema del presente trabajo de revisión. Informes de los primeros resultados del proyecto MetaHIT ${ }^{3}$ concluyen que el microbioma intestinal puede dividirse en tres grupos de poblaciones intestinales, que no están relacionados con la edad, el sexo, la nacionalidad o la dieta. El grupo de investigadores ha nombrado los enterotipos por el género dominante: Bacteroides, Prevotella y Ruminococcus (fig. 2). En el enterotipo 1 predominan las bacterias intesti- 
abla Ejemplo de cepas de probióticos en productos (adaptado de la OMGE) ${ }^{16}$

\begin{tabular}{|c|c|c|}
\hline Cepa (designaciones alternativas) & Nombre de marca & Fabricante \\
\hline Bifidobacterium animalis DN 13010 & Activia & Danone Dannon \\
\hline Bifidobacterium animalis subsp. lactis Bb-12 & & . Hansen \\
\hline Bifidobacterium breve akult & Bifiene & akult \\
\hline Bifidobacterium infantis 35624 & Align & Procter Gamble \\
\hline Bifidobacterium lactis HN019 (DR10) & Howaru'Mifido & Danisco \\
\hline \multicolumn{2}{|l|}{ Bifidobacterium longum BB536 } & Morinaga Milk Industry \\
\hline Enterococcus LAB SF 68 & Bioflorin & erbios-Pharma \\
\hline Escherichia coli Nissle 11 & Mutaflor & \\
\hline Lactobacillus acidophilus LA-5 & & Ardeypharm \\
\hline Lactobacillus acidophilus N FM & & hr. Hansen \\
\hline Lactobacillus casei DN-114 001 & & Danisco \\
\hline Lactobacillus casei CRL431 & Actimel, DanActive & Danone Dannon \\
\hline Lactobacillus casei F1 & & . Hansen \\
\hline Lactobacillus casei Shirota & ultura & Arla Foods \\
\hline Lactobacillus johnsonii Lal (Ljl) & akult & akult \\
\hline Lactobacillus lactis LIA & L 1 & Nestlé \\
\hline Lactobacillus plantarum 2 & Norrmejerier GoodBelly, Pro iva & NexFoods Probi \\
\hline Lactobacillus reuteri ATTC 55730 & Retueri & BioGaia Biologics \\
\hline Lactobacillus rhamnosus ATCC 53013 (LGG) & Vifit y otros & alio \\
\hline Lactobacillus rhamnosus LB21 & erum & Norrmejerier \\
\hline \multicolumn{3}{|l|}{ Lactobacillus salivarius UCC118 } \\
\hline Saccharomyces cerevisiae (boulardii) lio & DiarSafe, Ultralevure y otros & ren Laboratories, Biocodex y otros \\
\hline Analizado como mezcla: & Bio $\mathrm{K}+$ & Bio $\mathrm{K}+$ International \\
\hline \multirow{2}{*}{\multicolumn{3}{|c|}{$\begin{array}{l}\text { Lactobacillus acidophilus CL1285 y Lactobacillus } \\
\text { casei Lbc } 80 \text { r } \\
\text { Analizado como mezcla: }\end{array}$}} \\
\hline & & \\
\hline $\begin{array}{l}\text { Lactobacillus rhamnosus GR-1 y Lactobacillus } \\
\text { reuteri RC-14 }\end{array}$ & FemDophilus & . Hansen \\
\hline \multicolumn{3}{|l|}{ Analizado como mezcla: } \\
\hline $\begin{array}{l}\text { VSL \# } 3 \text { (mezcla de una cepa de Streptococcus } \\
\text { thermophilus, cuatro Lactobacillus spp. y tres } \\
\text { cepas de Bifidobacterium spp. }\end{array}$ & SL 3 & Sigma-Tau Pharmaceuticals, Inc. \\
\hline \multicolumn{3}{|l|}{ Analizado como mezcla: } \\
\hline \multirow{2}{*}{\multicolumn{3}{|c|}{$\begin{array}{l}\text { Lactobacillus acidophilus CUL60 y } \\
\text { Bifidobacterium bifidum CUL } 20\end{array}$}} \\
\hline & & \\
\hline \multicolumn{3}{|l|}{ Analizado como mezcla: } \\
\hline $\begin{array}{l}\text { Lactobacillus helveticus R0052 y Lactobacillus } \\
\text { rhamnosus R0011 }\end{array}$ & A Biotica y otros & Institut Rosell \\
\hline Analizado como mezcla: & & \\
\hline Bacillus clausii cepas O , NR. SIN, y T & Enterogermina & Sanofi-Aventis \\
\hline
\end{tabular}

nales del género Bacteroides, que además, según se observó, forman una comunidad bien diferenciada de flora intestinal junto con otras especies bacterianas. El enterotipo 2 está dominado por las bacterias Prevotella, y el enterotipo 3 está formado principalmente por Ruminococcus, aunque también contiene especies bacterianas como Staphylococcus y Gordonibacter, el más común. Según los investigadores del estudio, dichos grupos bacterianos pueden proporcionar, mediante la descomposición de materiales que llegan al intestino, energía y otros nutrientes que el ser humano podría aprovechar. abe destacar, por ejemplo, el caso del enterotipo 3, especializado en la descomposición de la mucina, un tipo de proteína producido por las células del epitelio que, a su vez, produce secreciones de mucosas en el organismo. Se cree que la descomposición de la mucina, por ejemplo, ayuda al intestino a absorber nutrientes beneficiosos para el cuerpo. Los tres enterotipos producen vitaminas. oncretamente, el enterotipo 1 produce vitamina B (biotina), $B_{2}$ (riboflavina) y C (ácido ascórbico), mientras que el 2 produce vitamina $B_{1}$ (tiamina) y ácido fólico. Gracias a sus comunidades de bacterias y a las diversas funciones que realizan, cada enterotipo se puede relacionar con una manera distinta de producir energía adaptada específicamente al organismo del huésped. Las formaciones bacterianas podrían incluso colaborar con el organismo huésped en distintos niveles e influir, en sinergias 
abla Principales especies aisladas de las diferentes regiones del tracto gastrointestinal (adaptado de Goldin et $\mathrm{al}^{20}$ )

\begin{tabular}{lll}
\hline Sitio & omposición & $\begin{array}{l}\text { Microorganismos } \\
(\mathrm{n} / \mathrm{ml})\end{array}$ \\
\hline Estómago & Streptococcus, & $10-100$ \\
& Lactobacillus \\
Duodeno y yeyuno & Similar al estómago & $10^{2}-10^{4}$ \\
Ileocecal & Bacteroides, & $10^{5}-10^{8}$ \\
& Clostridium, & \\
& Streptococcus, \\
olon & Lactobacillus \\
& Bacteroides, & $10^{11,5}-10^{12}$ \\
& Clostridium, \\
& Eubacterium, \\
& Pectococcus, \\
& Bifidobacterium, \\
& Streptococcus, \\
& Fusobacterium \\
\hline
\end{tabular}

con otros procesos, en la salud del individuo. Estos hallazgos, obtenidos mediante análisis de laboratorio y modelos celulares, son sin lugar a dudas el primer eslabón hacia un asesoramiento médico y dietético preventivo y personalizado, por lo que es posible la introducción de los probióticos como alimentos funcionales para regular la microbiota intestinal específicamente para lograr un efecto particular deseado. omplementariamente a los resultados del proyecto MetaHIT, diferentes estudios independientes en animales ${ }^{21}$ y revisiones de la literatura científica que incluyen estudios in vitro y en modelos animales refuerzan la hipótesis de que hay una posible asociación entre la microbiota intestinal, la regulación del peso corporal y las enfermedades asociadas a la obesidad, dada su posible influencia en las funciones metabólicas ${ }^{22,23}$ e inmunológicas del hospedador $^{24}$. Dichos resultados indican además que los desequilibrios en el ecosistema intestinal (disbiosis) y la endotoxemia (presencia de endotoxina en sangre) podrían ser factores inflamatorios relacionados con el desarrollo de resistencia a la insulina y del aumento del peso corporal.

La relación entre la composición de la microbiota intestinal y la salud humana ha llevado al diseño de estrategias dietéticas destinadas a favorecer la prevalencia de bacterias beneficiosas que mejoran el estado de salud. Raoult et $\mathrm{al}^{2}$ (2009) apuntan como hipótesis que puede haber conexión entre los probióticos y la obesidad. El aumento en la abundancia relativa de firmicutes y las reducciones proporcionales en la abundancia de bacteroidetes podrían ser el mecanismo que explicaría las diferencias halladas entre obesos y delgados ${ }^{2}$. En otros estudios sobre la microbiota en modelos animales que han comparado a individuos genéticamente obesos y flacos, también se ha establecido relación entre la obesidad y una mayor proporción de Archaea ${ }^{23}$. Asimismo, se ha detectado una reducción de Bifidobacterium y un aumento de Halomonas y Sphingomonas en la microbiota intestinal de animales genéticamente obesos ( $\mathrm{fa} / \mathrm{fa}$ ), en comparación con la de los sujetos de control ${ }^{25}$.
Algunos estudios muestran que las alteraciones en la composición de la microbiota intestinal asociadas a la obesidad (genética o inducida por la dieta) en animales pueden revertirse mediante la transferencia por vía oral de la microbiota intestinal de sujetos delgados ${ }^{26,27}$ o mediante la administración de prebióticos, por lo menos a corto plazo ${ }^{28}$. Asimismo, un estudio publicado en 2011 y financiado en parte a través del proyecto BIOCLA (Producción de derivados lácteos enriquecidos con LA ácido linoleico conjugado por métodos naturales) ha concluido que la administración de determinados Lactobacillus capaces de sintetizar CLA pueden modular la fisiología de las células adiposas de modelos animales y cambiar su composición significativamente. Pese a que los efectos del LA en humanos son poco convincentes $^{2}$, dichos hallazgos ponen de manifiesto que la ingesta de bacterias vivas puede tener algún efecto en la actividad metabólica de partes distantes del organismo ${ }^{30}$. Estos resultados estimulan a plantear la hipótesis de que la manipulación intencionada de la microbiota intestinal a través de la dieta podría ser una herramienta para prevenir o modificar el riesgo de obesidad y, en particular, las enfermedades metabólicas asociadas. Sin embargo, como bien advierten otros autores, esos hallazgos no pueden trasladarse al ser humano ni puede establecerse una relación causal mediante estudios en modelos celulares y animales $^{31,32}$, sino que se requieren muchos estudios clínicos realizados en humanos. En este sentido, cabe destacar que los cambios en las proporciones relativas entre firmicutes y bacteroidetes de la microbiota intestinal también se ha observado en humanos obesos ${ }^{33}$. Además, en dicho estudio clínico, los humanos obesos, tras seguir una dieta hipocalórica (baja en hidratos de carbono o en grasas), mostraron incrementos significativos en las proporciones de bacteroidetes paralelos a la pérdida de peso durante un periodo de intervención de $1 \mathrm{año}^{33}$. Estudios posteriores, realizados en un número más representativo de adolescentes obesos sometidos a tratamiento multidisciplinario para combatir la obesidad basado en una dieta con restricción calórica y un aumento de la actividad física, también han confirmado la existencia de relación entre la composición de la microbiota intestinal y el peso corporal ${ }^{34}$. En dicho estudio, la proporción relativa de los grupos Clostridium histolyticum, C. lituseburense y Eubacterium rectale-C. coccoides se redujo, mientras que la de los grupos Bacteroides-Prevotella y Lactobacillus aumentó tras la intervención en los sujetos que sufrieron una reducción de peso significativa. Asimismo, la composición de la microbiota del individuo pareció influir en la pérdida de peso durante la intervención ${ }^{34}$. En otros estudios, en los que se ha investigado la microbiota de gemelos obesos y delgados, también se ha asociado una menor proporción de bacteroidetes y mayor de actinobacterias a la obesidad ${ }^{35}$. Un estudio llevado a cabo por Ilmonen et $\mathrm{al}^{36}$ (2009) concluye que las mujeres embarazadas que toman suplementos probióticos (Lactobacillus y Bifidobacterium) a partir del primer trimestre de gestación tienen menos propensión a la obesidad central (definida como IMC $\geq 30$ o circunferencia de cintura $>80 \mathrm{~cm}$ ). Este es el primer estudio que establece una asociación entre la dieta complementada con probióticos durante el embarazo y que la lactancia influye en la adiposidad femenina durante los 12 meses posparto. Así, Ilmonen et al señalan que el consumo de 


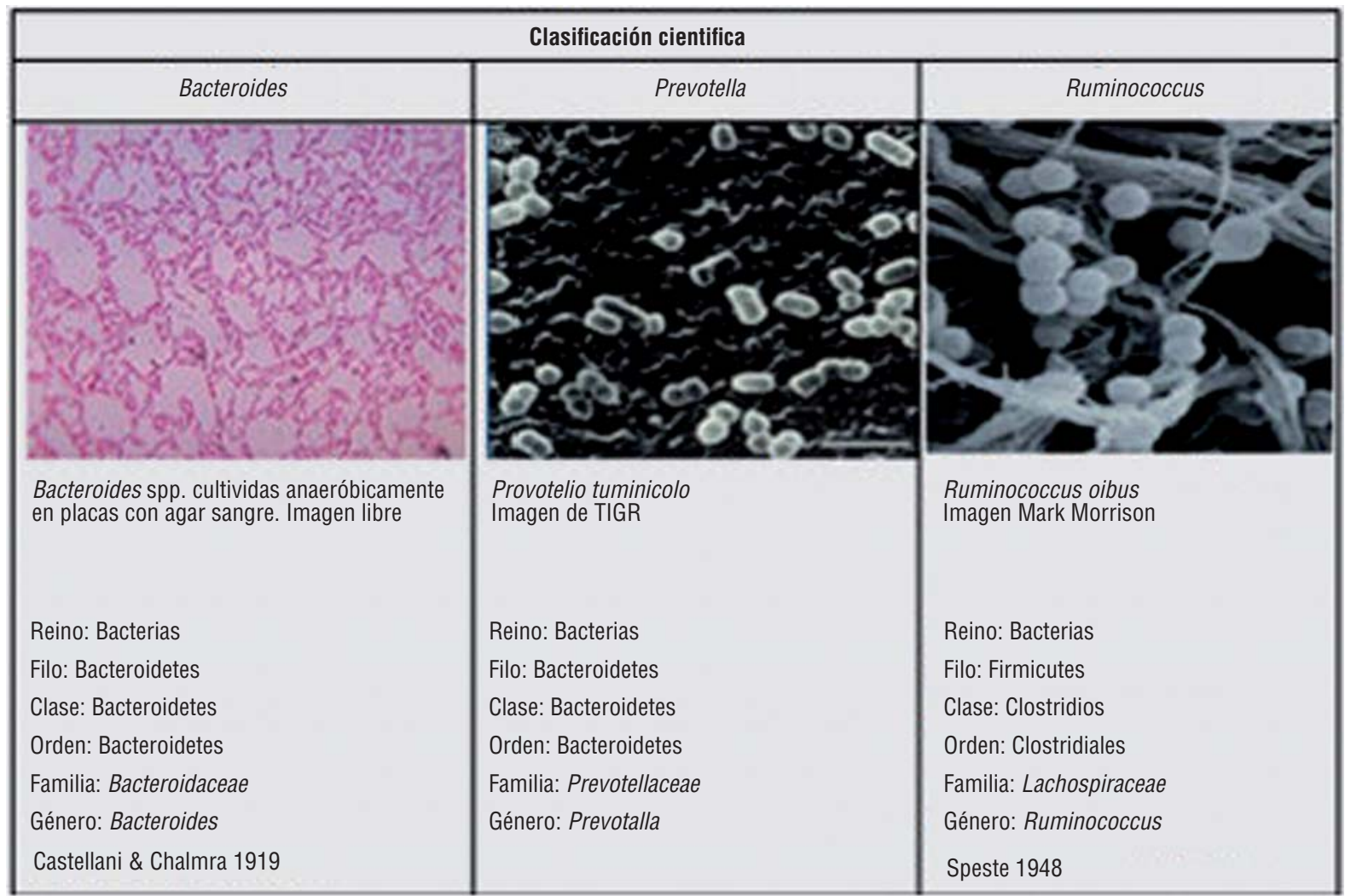

i ura Clasificación científica de los géneros dominantes en la flora intestinal de las personas.

probióticos junto con una dieta equilibrada es un método razonablemente económico, práctico, seguro y potencialmente satisfactorio para usarlo con otros factores de estilo de vida en el control de la adiposidad central.

No obstante, también se han realizado estudios de intervención con otros probióticos, con resultados negativos en el metabolismo de los lípidos (tabla 3$)^{3}$. Así por ejemplo, Raoult et $\mathrm{al}^{2}$ (2009) describen que algunos estudios han mostrado que Lactobacillus spp. se asocian con el aumento de peso en los niños tratados para la diarrea, y que los probióticos con L. rhamnosus pueden ocasionar aumento de peso en los niños independiente de la enfermedad para la que se prescribió ese probiótico. Por todo esto, y considerando la epidemia de obesidad infantil que se está produciendo en muchos países desarrollados, piden un completo estudio de los efectos de los probióticos en el peso corporal.

Por su parte, el Dr. Dusko, coordinador del proyecto MetaHIT-Francia, describe ${ }^{31}$ que Bifidobacterium spp. no pertenecen al filo de los firmicutes, sino al de actinobacterias (fig. 1) y que la asociación de firmicutes con la obesidad es aún polémica. Los firmicutes son un filo, grupo extraordinariamente diverso, que incluye microorganismos patógenos (Bacillus antharacis, relacionado con el ántrax), comensales en la flora humana (Faecalibacterium prausnitzii: su escasez se asocia con la enfermedad de rohn), así como las bacterias utilizadas para la elaboración del queso o el yogur, tales como L. delbrueckii subsp. bulgaricus. El autor quiere advertir que es un error generalizar habiendo tomado un eslabón alto en la clasificación científica.

\section{Discusión y conclusiones}

El futuro de la alimentación funcional con probióticos va orientado, ante todo, a la mejora de la salud a través de la prevención de ciertas enfermedades o factores de riesgo como el colesterol, la presión arterial alta o la obesidad. Hay gran variedad de estudios, en su mayoría realizados en animales y modelos celulares, que muestran una relación estrecha entre el microbioma y los efectos en el metabolismo energético y asimismo sobre una de las causas de la génesis de la obesidad. Pese a que este tipo de estudios no es apropiado para predecir la funcionalidad de los microorganismos probióticos en el intestino humano, gran parte de las hipótesis resultantes coinciden en indicar que algunas mezclas de microorganismos de la flora intestinal podrían proteger de la obesidad al ser humano, además de ejercer efectos fisiológicos sistémicos relacionados con efectos beneficiosos en el metabolismo lipídico y diversos factores de riesgo de enfermedad cardiovascular. No obstante, la mayoría de los ensayos clínicos en humanos se han realizado con cohortes muy pequeñas, y otros como el MetaHIT aún se encuentra en desarrollo. Aunque se necesitan más estudios 
abla Efectos de los probióticos en la obesidad y las alteraciones metabólicas en humanos ${ }^{3}$

\begin{tabular}{|c|c|c|c|c|}
\hline $\begin{array}{l}\text { Probiótico prebiótico } \\
\text { (dosis/día) }\end{array}$ & Sujetos duración & Diseño & Resultado & Referencia \\
\hline \multirow[t]{2}{*}{$\begin{array}{l}\text { L. plantarum } 299 v(5 \times 10 \text { ufc } \\
\text { día) }\end{array}$} & $\begin{array}{l}\text { Sujetos } \\
\text { hipercolesterolémicos }\end{array}$ & , R, D & Reducción de CLDL en suero & \multirow[t]{2}{*}{38,39} \\
\hline & $\begin{array}{l}\text { Fumadores } \\
6 \text { semanas }\end{array}$ & , R, D & $\begin{array}{l}\text { Reducción de presión sistólica, } \\
\text { leptina y fibrinógeno }\end{array}$ & \\
\hline L. acidophilus $145\left(10^{6}-10^{8}\right.$ ufc g), & Mujeres; 15 con & 0 & Aumento de cHDL y & 40 \\
\hline $\begin{array}{l}10^{5} \text { ufc/g) y } 1 \% \text { oligofructosa en } \\
\text { yogur con } \mathrm{S} \text {. thermophilus y }\end{array}$ & $\begin{array}{l}\text { y } 14 \text { con } \\
\text { hipercolesterolemia }\end{array}$ & & plasma & \\
\hline Lactococcus lactis (300 g/día) & $\begin{array}{l}\text { Tres periodos de } \\
\text { semanas: } 1 \text {. control para } \\
\text { todas; } 2 \text {. y } 3 \text {. intercambio } \\
\text { control problema }\end{array}$ & & Colesterol total y cLDL (NS) & \\
\hline $\begin{array}{l}\text { L. acidophilus DDS-1 y B. longum } \\
\text { UABL-14 (109 UFC/cápsula) con } \\
\text { oligofructosa 10-15 mg por } \\
\text { cápsula, } 3 \text { cápsulas día }\end{array}$ & $\begin{array}{l}\text { Sujetos } \\
\text { normocolesterolémicos } \\
2 \text { meses o } 2 \text { ciclos } \\
\text { menstruales }\end{array}$ & , R, S & $\begin{array}{l}\text { olesterol total, cHDL, cLDL y } \\
\text { triglicéridos (NS) }\end{array}$ & 42 \\
\hline Oligofructosa (16 g/día) & $\begin{array}{l}\text { Sujetos sanos con } \\
\text { normopeso } \\
2 \text { semanas }\end{array}$ & , R, S & $\begin{array}{l}\text { Aumento de saciedad después } \\
\text { del desayuno y la cena } \\
\text { Reducción de hambre y } \\
\text { consumo de comida después de } \\
\text { la cena }\end{array}$ & 43 \\
\hline Oligofructosa (8 g/día) & $\begin{array}{l}\text { Sujetos sanos con } \\
\text { normopeso } \\
12 \text { meses }\end{array}$ & & $\begin{array}{l}\text { Reducción de IM, z-score del } \\
\text { IM y grasa total }\end{array}$ & 44 \\
\hline
\end{tabular}

para evaluar los efectos de los probióticos en la incidencia de obesidad, si las relaciones establecidas entre la microbiota intestinal, el metabolismo y la inmunidad se demostraran mediante ensayos clínicos en humanos, el uso de estrategias dietéticas destinadas a modular la composición de la microbiota basadas en probióticos podría contribuir al control de los trastornos metabólicos de forma más eficaz y se abrirían nuevas puertas de estudio para obtener un mejor conocimiento de la biología humana y numerosas aplicaciones en la salud y la nutrición humana.

\section{A radecimientos}

Los autores agradecen los valiosos aportes del Dr. Francisco Guarner, responsable del proyecto MetaHIT de la Unidad de Investigación de Aparato Digestivo, Hospital Universitario all d Hebron, Barcelona, España.

\section{Conflictos de intereses}

Ninguno.

\section{iblio raf a}

1. Basulto ,Manera M, Baladia E. Postura del GREP-AEDN: la obesidad como enfermedad. Act Diet. 2008;12:98-9.

2. Raoult D. Probiotics and obesity: a link Nat Rev Microbiol. 2009;7:616.

3. Arumugam M, Raes , Pelletier E, et al. Enterotypes of the human gut microbiome. Nature. 2011;473:174-80.

4. Ortiz H, Galán I, Martín López R, et al. Prevalencia de sobrepeso y obesidad y efectos en la mortalidad atribuible en la comunidad de Madrid. Bol Epidemiol om Madrid. 2010; 16(6).

5. Ministerio de Sanidad y Política Social - Campaña 2007. Situación actual y tendencia en España Internet actualizado 23 Oct 200 . Disponible en: http: www.msps.es campannas campanas06/obesidadlnfant3.htm

6. Aranceta-Bartrina J, Serra-Majem L, Foz-Sala M, et al. Prevalence of obesity in Spain. Med Clin (Barc). 2005;125:460-6.

. Banegas R, Lopez-Garcia E, Gutierrez-Fisac L, et al. A simple estimate of mortality attributable to excess weight in the European Union. Eur J Clin Nutr. 2003;57:201-8.

8. Zugasti Murillo A, Moreno Esteban B. Obesidad, factor de riesgo cardiovascular. Rev Esp Obes. 2005;3:89-94.

. Salas-Salvadó , Rubio MA, Barbany M, et al. onsenso SEEDO 200 para la evaluación del sobrepeso y la obesidad y el esta- 
blecimiento de criterios de intervención terapéutica. Med lin (Barc). 2007;128:184-96.

10. Ashwell M. oncepto sobre los alimentos funcionales. Ilsi Europe oncise Monograph Series edición electrónica 2002. Disponible en: http: www.argenbio.org adc uploads pdf alimentosfuncionalesiLSI.pdf

11. Holzapfel WH, Haberer P, Snel J, et al. Overview of gut flora and probiotics. Int J Food Microbiol. 1998;41:85-101.

12. Klein G, Pack A, Bonaparte C, et al. Taxonomy and physiology of probiotic lactic acid bacteria. Int Food Microbiol. 1998;41:103-25

13. FAO/OMS 2006. Probióticos en los alimentos. Estudio FAO Alimentación y Nutrición 85. Disponible en: ftp://ftp.fao.org/ docrep/fao/009/a0512s/a0512s00.pdf

14. Collins JK, Thornton G, O'Sullivan GO. Selection of probiotic strains for human applications. Int Dairy J. 1998;8:487-90.

15. Morelli L. In vitro selection of probiotic lactobacilli: A critical appraisal. Curr Issues Intestinal Microbiol. 2000;1:59-67.

16. Guarner F, Khan Aamir G, Garisch J, et al. Equipo de Revisión. Guías prácticas: probióticos y prebióticos. Organización Mundial de Gastroenterología; 2008.

1. Aguilera Garca M, Barberá Mateos M, Diaz Ligia E, et al. Alimentos funcionales. Aproximación a una nueva alimentación. Inutcam 2008. Dirección General de Salud Pública y Alimentación

18. Tannock GW. Analysis of the intestinal microflora A renaissance. Antonie Van Leeuwenhoek. 1999;76:265-78.

19. Mitsuoka T. Intestinal flora and aging. Nutr Rev. 1992;50:438-46.

20. Goldin BR. Intestinal microflora: Metabolism of drugs and carcinogens. Ann Med. 1990;22:43-8.

21. Cani PD, Bibiloni R, Knauf $C$, et al. Changes in gut microbiota control metabolic endotoxemia- induced inflammation in highfat diet-induced obesity and diabetes in mice. Diabetes. 2008;57:1470-81.

22. B ckhed F, Ding H, ang $\mathrm{T}$, et al. The gut microbiota as an environmental factor that regulates fat storage. Proc Natl Acad Sci U S A. 2004;101:15718-23.

23. Ley RE, B ckhed F, Turnbaugh $P$, et al. Obesity alters gut microbial ecology. Proc Natl Acad Sci U S A. 2005;102:11070-5.

24. Mazmanian SK, Liu CH, Tzianabos AO, Kasper DL. An immunomodulatory molecule of symbiotic bacteria directs maturation of the host immune system. Cell. 2005;122:107-18.

25. Waldram A, Holmes E, Wang Y, et al. Top-down systems biology modeling of host metabotype- microbiome associations in obese rodents. J Proteome Res. 2009;8:2361-75.

26. Turnbaugh PJ, Ley RE, Mahowald MA, et al. An obesity-associated gut microbiome with increased capacity for energy harvest. Nature. 2006;444:1027-31.

2 . Turnbaugh $P, B$ ckhed F, Fulton $L$, et al. Diet-induced obesity is linked to marked but reversible alterations in the mouse distal gut microbiome. Cell Host Microbe. 2008;3:213-23.

28. Cani PD, Neyrinck AM, Fava F, et al. Selective increases of bifidobacteria in gut microflora improves high-fat-diet-induced diabetes in mice through a mechanism associated with endotoxaemia. Diabetologia. 2007;50:2374-83.

29. Onakpoya IJ, Posadzki PP, Watson LK, et al. The efficacy of long-term conjugated linoleic acid (CLA) supplementation on body composition in overweight and obese individuals: a systematic review and meta-analysis of randomized clinical trials. Eur J Nutr. 2012;51:127-34.

30. Rosberg- ody E, Stanton, O Mahony L, et al. Recombinant lactobacilli expressing linoleic acid isomerase can modulate the fatty acid composition of host adipose tissue in mice. Microbiology. 2011;157(Pt 2):609-15.

31. Dusko Ehrlich S. Probiotics- little evidence for a link to obesity. Nat Rev Microbiol. 200 ; : 01; autor reply 01.

32. Delzenne N, Reid G. No causal link between obesity and probiotics. Nat Rev Microbial. 200 ; : 01; author reply 01.

33. Ley RE, Turnbaugh PJ, Klein S, et al. Microbial ecology: human gut microbes associated with obesity. Nature. 2006;444: 1022-3.

34. Santacruz A, Collado MC, Azcona C, et al. Weight loss influences gut microbial composition in overweight adolescents. Obesity. $200 ; 23: 1-10$

35. Turnbaugh PJ, Hamady M, Yatsunenko T, et al. A core guts microbiome in obese and lean twins. Nature. 2009;457:480-4.

36. Ilmonen J, Isolauri E, Poussa T, et al. Impact of dietary counselling and probiotic intervention on maternal anthropometric measurements during and after pregnancy: A randomized placebo-controlled trial. Clin Nutr. 2011;30:156-64.

37. Sanz Y, Santacruz A, Dalmau J. Influencia de la microbiota intestinal en la obesidad y las alteraciones del metabolismo. Acta Pediatr Esp. 2009;67:437-42.

38. Bukowska H, Pieczul-Mróz J, Jastrzebska M, Chelstowski K, Naruszewicz $M$. Decrease in fibrinogen and LDL-cholesterol levels upon supplementation of diet with Lactobacillus plantarum in subjects with moderately elevated cholesterol. Atherosclerosis. 1998;137:437-8.

39. Naruszewicz M, Johansson ML, Zapolska-Downar D, Bukowska H. Effect of Lactobacillus plantarum 2 v on cardiovascular disease risk factors in smokers. Am J Clin Nutr. 2002;76:1249-55.

40. Kiessling G, Schneider J, Jahreis G. Long-term consumption of fermented dairy products over 6 months increases HDL cholesterol. Eur J Clin Nutr. 2002;56:843-9.

41. Simons LA, Amansec SG, Conway P. Effect of Lactobacillus fermentum on serum lipids in subjects with elevated serum cholesterol. Nutr Metab Cardiovasc Dis. 2006;16:531-5.

42. Greany KA, Bonorden MJ, Hamilton-Reeves JM, McMullen MH, Wangen KE, Phipps WR, et al. Probiotic capsules do not lower plasma lipids in young women and men. Eur J Clin Nutr. 2008;62:232-7.

43. Cani PD, Joly E, Horsmans Y, Delzenne NM. Oligofructose promotes satiety in healthy human: a pilot study. Eur lin Nutr. 2006;60:567-72.

44. Abrams SA, Griffin IJ, Hawthorne KM, Ellis KJ. Effect of prebiotic supplementation and calcium intake on body mass index. Pediatr. 2007;151:293-8. 